\title{
Hyaluronan turnover and hypoxic brown adipocytic differentiation are co-localized with ossification in calcified human aortic valves
}

Elizabeth H. Stephens, MD, PhD ${ }^{1}$, Jerome G. Saltarrelli Jr, PhD ${ }^{1}$, Liezl R. Balaoing, BS ${ }^{1}$, L. Scott Baggett, PhD $^{2}$, Indrajit Nandi, BS ${ }^{1}$, Kristin M. Anderson, BS ${ }^{1}$, Joel D. Morrisett, PhD $^{3}$, Michael J. Reardon, MD $^{4}$, Melanie A. Simpson, PhD $^{5}$, Paul H. Weigel, PhD $^{6}$, Elizabeth A.

Olmsted-Davis, $\mathbf{P h D}^{7}$, Alan R. Davis, $\mathrm{PhD}^{7}$, and K. Jane Grande-Allen, $\mathrm{PhD}^{1}$

${ }^{1}$ Department of Bioengineering, Rice University, Houston, TX 77005

${ }^{2}$ Department of Statistics, Rice University, Houston, TX 77005

${ }^{3}$ Departments of Medicine and Biochemistry, Baylor College of Medicine, Houston, TX

${ }^{4}$ Department of Cardiovascular Surgery, The Methodist Hospital, Houston, TX 77030

${ }^{5}$ Department of Biochemistry, University of Nebraska, Lincoln, NE 68588

${ }^{6}$ Department of Biochemistry and Molecular Biology, Oklahoma University Health Science Center, Oklahoma City, OK 73104

${ }^{7}$ Center for Cell and Gene Therapy, Baylor College of Medicine, Houston, TX, 77030

\section{Abstract}

The calcification process in aortic stenosis has garnered considerable interest but only limited investigation into selected signaling pathways. This study investigated mechanisms related to hypoxia, hyaluronan homeostasis, brown adipocytic differentiation, and ossification within calcified valves. Surgically explanted calcified aortic valves $(n=14)$ were immunostained for markers relevant to these mechanisms and evaluated in the center (NodCtr) and edge (NodEdge) of the calcified nodule (NodCtr), tissue directly surrounding nodule (NodSurr); center and tissue surrounding small "prenodules" (PreNod, PreNodSurr); and normal fibrosa layer (CollFibr). Pearson correlations were determined between staining intensities of markers within regions. Ossification markers primarily localized to NodCtr and NodEdge, along with markers related to hyaluronan turnover and hypoxia. Markers of brown adipocytic differentiation were frequently colocalized with markers of hypoxia. In NodCtr and NodSurr, brown fat and ossification markers correlated with hyaluronidase-1, whereas these markers, as well as hypoxia, correlated with hyaluronan synthases in NodEdge. The protein product of tumor necrosis factor-a stimulated gene-6 strongly correlated with ossification markers and hyaluronidase in the regions surrounding the nodules (NodSurr, PreNodSurr). In conclusion, this study suggests roles for hyaluronan homeostasis and the promotion of hypoxia by cells demonstrating brown fat markers in calcific aortic valve disease.

\footnotetext{
C 2012 Elsevier GmbH. All rights reserved.

Address for correspondence: K. Jane Grande-Allen, Ph.D., Department of Bioengineering, Rice University, 6100 Main St., MS 142, Houston, TX 77005, Phone: 713-348-3704, Fax: 713-348-5877, grande@ rice.edu.

Publisher's Disclaimer: This is a PDF file of an unedited manuscript that has been accepted for publication. As a service to our customers we are providing this early version of the manuscript. The manuscript will undergo copyediting, typesetting, and review of the resulting proof before it is published in its final citable form. Please note that during the production process errors may be discovered which could affect the content, and all legal disclaimers that apply to the journal pertain.
} 


\section{Keywords}

brown adipocytes; hyaluronan; hypoxia; calcification; aortic valve

\section{Introduction}

Valve disease is widely prevalent in our society, with valve replacement or repair in almost 100,000 people in the United States each year [16]. The most common heart valve disease is calcific aortic valve disease (CAVD), also known as calcific aortic stenosis. CAVD is associated with aging, obesity, and metabolic syndrome [12], but there are no treatments for CAVD other than surgical aortic valve replacement, nor are there any medications that specifically target CAVD. Moreover, investigators have only begun to explore possible mechanisms for the progression of CAVD in the last several years.

Several previous studies of the development and progression of CAVD have related to the deposition of calcific nodules, which is a hallmark of the advanced valvular sclerotic lesion, and which cause the leaflets to become stiff and the valve stenotic. These nodules, which may appear as hydroxyapatite crystals and show characteristics of heterotopic bone [19], are found in association with lipids both in human valves and in animal models. Calcified leaflets also contain osteoblast-like cells and an abundance of several osteogenic mediators, including bone morphogenic protein-2 (BMP-2) [21]. Investigations of heterotopic bone formation in a mouse muscle model [23] have shown associations between overproduction of BMP-2, rapid production of brown adipocytes that stimulate hypoxic conditions, and heterotopic ossification. In this model, three days after the delivery of excess BMP-2 to the muscle, gene expression was strongly upregulated for several markers that are also reportedly elevated in either atherosclerosis or CAVD, including CD44, E-selectin, apolipoprotein E, cycloxygenase-2, prostaglandins, and the small proteoglycan decorin [21]. Expression of many of these markers is regulated by the inflammatory cytokine tumor necrosis factor- $a$ (TNF- $a$ ), which is intriguing because the protein product of TNF- $a$ stimulated gene-6 (TSG-6) provides a mechanistic link between BMP-2 and the glycosaminoglycan hyaluronan (HA).

Due to the complex ability of HA to bind to lipids and monocytes, the many regulators of HA homeostasis (synthases, receptors, and degrading enzymes) are associated with the progression of atherosclerosis and vascular injury [6]. These factors may impact the progression of CAVD as well, but have not been previously investigated in calcified aortic valves. It has recently been shown, however, by our group [29] and Johansson et al. [11] that the abundance of HA varies substantially between large heavily calcified nodules, diffuse and smaller calcified nodules, and normal-appearing regions of stenotic aortic valves. HA and BMP-2 can also bind to TSG-6 through the same Link-protein-like domain; TSG-6binding of BMP-2 inhibits ossification by mesenchymal stem cells [32]. When HA is present and bound to the TSG-6 Link domain, TSG-6 cannot inhibit the effects of BMP-2. Perhaps for this reason, HA has been shown to be a very efficient carrier of BMP-2 promoting the mineralization of tissue engineered bone and bony ingrowth into implants [10].

The purpose of this study was to investigate the relationships between BMP-2, hypoxia, HA regulation, and ossification by performing immunohistochemistry on calcified aortic valves. Although HA regulation has been widely studied in heart valve embryonic development [13, 24], there has been no report of these factors in the context of normal or diseased aortic valves of adults. In addition, the hypoxia-brown adipocyte relationship has not been previously investigated in heart valves. The two primary hypotheses examined in this study 
were first that BMP-2-associated hypoxia, as demonstrated by markers of brown adipocytic differentiation, would be co-localized with markers for bone and chondrocytes. The second hypothesis was that there would be distinct regions within the leaflet demonstrating strong expression of markers for either HA synthesis or HA degradation, and that these markers would be co-localized with expression of mechanisms of hypoxia, brown adipocytic differentiation, and ossification.

\section{Materials and methods}

\section{Tissue procurement and decalcification}

Diseased aortic valves removed during valve replacement surgery were determined by surgeons and pathologists as calcified with no sign of rheumatic disease. These tissues were obtained from the Methodist DeBakey Heart and Vascular Center of the Methodist Hospital (Houston, TX) and the Cooperative Human Tissue Network (CHTN) (n=14, mean age $65 \pm 15,80 \%$ male). At Methodist, patients with aortic insufficiency were considered eligible for aortic valve replacement if they had ejection fractions $<55 \%$ and an end-systolic left ventricular diameter $>55 \mathrm{~mm}$. Patients with aortic stenosis were eligible for aortic valve replacement only if they were symptomatic and demonstrated any of the following characteristics: aortic valve area $<1.0 \mathrm{~cm} 2$ or $<0.6 \mathrm{~cm} 2 / \mathrm{m} 2$ (normalized to body surface area); echocardiographic flow velocity $>4 \mathrm{~m} / \mathrm{sec} 2$, or transvalvular gradient $>50 \mathrm{mmHg}$. Comparable inclusion criteria were employed for valves obtained through CHTN. Study authors KJGA and JDM obtained approval from the Institutional Review Boards at Rice University and Baylor College of Medicine for the research use of these tissues. The 10 calcified aortic valves obtained from Methodist were selected at random from a group of surgical specimens collected for analysis by study author JDM. The only defining characteristic of the selected valves was having at least 1-2 intact leaflets, as opposed to having been surgically resected only as calcified leaflet fragments. These valves were either stored in $50 \%$ glycerol in phosphate-buffered saline (PBS) at $-20^{\circ} \mathrm{C}$ or in RNAlater (Applied Biosystems, Foster City, CA) at $-80^{\circ} \mathrm{C}$. Prior to histological processing, glycerol or RNAlater was removed by dialyzing overnight in fresh PBS. Afterwards, several 2-3 millimeter radial strips were cut from each leaflet and placed into $10 \%$ formic acid at room temperature for 12-24 hours (depending on level of calcification) to decalcify the sections allowing routine paraffin embedding; it has been previously shown that formic acid treatment decalcifies tissues without damaging antigenicity [1]. After a palpable level of decalcification was achieved, diseased valves were embedded in paraffin and cut into multiple serial sections according to routine procedures. The 4 specimens obtained from the CHTN were similarly decalcified, embedded in paraffin, and sectioned. The CHTN-stained tissues could not be distinguished from the samples from Methodist.

\section{Histology and immunohistochemistry}

Sections were stained with Movat pentachrome to demonstrate the general microstructure of the leaflet. Adjacent sections were also stained immunohistochemically for a range of intracellular, plasma membrane-associated, or extracellular proteins related to osteogenesis and chondrogenesis, hyaluronan homeostasis, and hypoxia (Table 1) using standard techniques as previously described [28]. All sections underwent citrate buffer-based antigen retrieval (Antigen Decloaker, Biocare Medical, Concord, CA) or enzymatic pre-treatment appropriate for each antibody. Positive staining was demonstrated by a chromogen reaction using Vectastain Elite ABC and diaminobenzidine kits (Vector Laboratories, Burlingame, CA). All samples were counterstained with hematoxylin. To minimize variability, multiple samples were taken from a given patient's valve. Negative controls for all markers were performed in the absence of the primary antibody. 
To evaluate co-localization of BMP-2, TSG-6, and UCP-1 with Hyal-1 several sections were double-stained using immunofluorescence. The samples were processed using citrate buffer based antigen retrieval and primary antibodies as described above. However, goat anti-rabbit Alexa-fluor 555 and donkey anti-mouse Alexa-fluor 633 secondary antibodies (Invitrogen, Carlsbad, CA) were used after primary antibody incubation. The detection of positive signal and colocalization on each sample was demonstrated by imaging the slides using a fluorescent microscope at each fluorophore channel. A DAPI nuclei stain was used as a fluorescent counterstain for each double-stained sample.

\section{Analysis of immunohistochemical staining}

Several regions of the singly-stained, radially oriented leaflet sections were evaluated to assess the mean 8-bit intensity of brown DAB chromagen staining using ImageJ software (NIH, Bethesda, MD). Note that the brown chromogen develops enzymatically with a nonlinear reaction curve, thus the data should be considered semi-quantitative. To determine the reproducibility of these mean intensity measurements, these several regions were measured on two separate occasions; it was calculated that, on average, the two sets of measurements of staining intensities differed by approximately $7 \%$. Calcified nodules were categorized as large and presumably mature ("nodule") or small and presumably early-stage ("prenodule"). Prenodules were identified as subsidiary, substantially smaller nodules (typically $₫ / 2$ of leaflet height), which were not continuous with the main nodule; prenodules were generally located closer to the annulus than was the main nodule. Regions of interest were identified (Fig. 1) in the nodule center (NodCtr, innermost 1/3), edge (NodEdge, outermost 1/3), and surrounding tissue (NodSurr); in the prenodule center (PreNod) and surrounding tissue (PreNodSurr); and in the fibrosa layer of a normal-appearing region of the same leaflet far away from the nodules (CollFibr). The precise locations of the analysis regions were noted on images of the Movat stained sections to ensure that the regions of immunohistochemical staining intensities were performed using the exact same locations on all of the serial tissue sections.

\section{Statistical analysis}

Statistical analysis was performed using SAS (Cary, NC). Correlations between staining intensities of different proteins within individual leaflet regions (to assess co-localization) were calculated using Pearson Product Moments. Multiple tissue sections from individual patients were managed using a repeated measure analysis. For correlations between intensities of different components, the level of significance was reduced to P $₫ .0045$ since 11 markers were being considered. Correlations for which $P \unlhd 0.01$ were considered trends.

\section{Results}

The large number of markers that were immunohistochemically evaluated and the dual nature of the results (visual and measured staining intensities) generated a substantial amount of data that will be presented as follows. The visual results will be reported first to describe the most common location (region within the valve) of the various markers, as well as their co-localization with other markers. Correlations between measured staining intensities of different markers will be described next, initially through a report of markers that would logically be expected to be found together because they are in the same mechanistic family. Subsequently, there will be a report of correlations between markers found in different mechanistic families. Whenever possible, these results will be grouped according to the different regions of the calcified valve leaflet. 


\section{Co-localizations between markers}

BMP-2 was primarily demonstrated within the calcific nodule (meaning both NodCtr and NodEdge, unless otherwise noted), with some staining in NodSurr as well. BMP-2 was often co-localized with staining for Hyal-1 and to a lesser extent HIF-1a (Figs. 2-3). HIF-1a staining was generally observed inside the nodule as well (whereas staining in NodCtr usually appeared stronger than in NodEdge), and was usually co-localized with S-100, BMP-2, and PGC-1a (Fig. 3). TSG-6 was often localized to the inside of the nodule (with PGC-1 $a$ and UCP-1), but could also be found in NodSurr; TSG-6 was partly co-localized with BMP-2 (Figs. 2-3). Similarly, Hyal-1 and HAS were most often demonstrated within the nodule, but were also found in NodSurr, where Hyal-1 was often co-localized with TSG-6. Cells staining positively for a-SMA were abundant surrounding the nodule (Fig. 2). Periostin was predominantly localized within and immediately adjacent to the nodule, more so than in the remainder of the leaflet tissue (Fig. 4).

\section{Correlations within mechanistic families}

There were numerous correlations among markers in the same mechanistic family, as expected (Table 2). For the family of regulators of HA, CD168 was significantly and very strongly associated with Hyal-1 in NodSurr; this same association, albeit less strong, was also a trend in CollFibr. HARE was also significantly associated with Hyal-1 in CollFibr. For the family of hypoxic regulators, HIF-1a was strongly associated with markers for brown fat differentiation (PGC-1a in NodEdge and UCP-1 in PreNodSurr). A less strong link between HIF-1a and UCP-1 was a trend in NodSurr. PGC-1a and UCP-1 were also associated in NodCtr. Finally, there were numerous associations between markers of chondrogenesis and ossification: BMP-2, S-100, and TSG-6 were all significantly associated with each other in NodSurr (Fig. 5). TSG-6 had trends of association with S-100 in NodEdge and BMP-2 in PreNodSurr. Periostin was also significantly and strongly associated with TSG-6 in NodCtr.

\section{Correlations between mechanistic families}

The findings of correlations between markers from different mechanistic families suggest that there are interactions between these agents, and that these interactions can be found in distinct regions of the leaflet (Table 3). Correlations between these markers were unique depending on the different regions, suggesting that the processes were influenced by their location relative to the nodule. In NodCtr, HA degradation (Hyal-1) strongly correlated with markers of brown fat differentiation (PGC-1a and UCP-1, Fig. 6A), and had a trend of association with chondrogenic differentiation (S-100). There were also correlations between chondrogenic/osteogenic differentiation and brown fat differentiation in this region: S-100 was significantly correlated with UCP-1, as was BMP-2 and PGC-1a. In contrast to NodCtr, in NodEdge, the marker for the enzymes for hyaluronan synthesis, HAS, was significantly associated with the brown fat marker PGC-1a and marker of hypoxia HIF-1a, as well as a trend of association with the ossification marker BMP2 (Fig. 7A). Chondrogenic differentiation and hypoxia were also linked by a moderate association between S-100 and UCP-1 in NodEdge (Fig. 7B). In this region, periostin demonstrated a trend of association with HA regulation (HAS), hypoxia (HIF-1a), and brown fat differentiation (PGC-1a).

Far more significant correlations were found between mechanistic families in NodSurr. Significant interactions between HA regulation, chondrogenesis, and ossification were demonstrated by correlations of BMP-2, TSG-6, and S-100 with Hyal-1 and CD168. Most notably, Hyal-1 showed strong correlations with the brown fat marker UCP-1 (Fig. 6A), as well as the ossification marker BMP2. Significant interactions between hypoxic regulation and HA regulation were also shown by correlations of UCP-1 with CD168. Links between UCP-1 and chondrogenesis were more moderate, with only a significant correlation with 
S-100. TSG-6 also demonstrated associations with BMP-2 and HYAL-1 (Figs. 2, 3, and 6B) in this region.

In PreNod and PreNodSurr, there were fewer correlations between mechanistic families, but the correlations were extremely strong. Within the PreNod, ossification and HA regulation showed strong associations with Hyal-1 strongly correlating with the markers S-100 and BMP-2 (Fig. 7C). PreNod also showed correlation trends associating HARE with S-100 and TSG-6 with Hyal-1. In PreNodSurr, Hyal-1 was significantly correlated with ossification markers BMP-2 and TSG-6 (Fig. 6B).

In CollFibr, several moderate correlations between mechanistic families were present. Hypoxic regulation (UCP-1) was significantly correlated with HA regulation (CD168, Hyal-1). Links between hypoxic regulation and ossification were also demonstrated by a significant correlation between PGC-1a and periostin, and a correlation trend between UCP-1 and S-100. Finally, HA regulation (HARE) was linked with ossification (S-100).

\section{Discussion}

This work demonstrates the presence of several markers of hypoxia and brown fat-driven remodeling mechanisms within calcified aortic valves, as well as mechanisms linked to hyaluronan balance and TSG-6, a molecule that can either promote or suppress BMP-2induced calcification depending on the presence or absence of HA. These results support the proposed hypotheses and also highlight the role of Hyal-1, which was strongly linked with both hypoxic and ossification markers. Aspects of brown fat differentiation and hyaluronan regulation have not been previously investigated in calcified aortic valves.

\section{Role for hypoxia and brown fat in calcification}

The demonstration of two markers signifying the differentiation of brown fat cells, UCP-1 and PGC-1a, that were highly correlated with the presence of HIF-1a within and surrounding the nodules of these calcified aortic valves provides new directions for investigation of disease mechanisms and novel therapies. In murine models of heterotopic ossification within muscle, brown adipocytes begin to accumulate within 24 hours of delivery of adenovirus transduced cells expressing BMP2 [4, 23]. These brown adipocytes generated hypoxic stress within the muscle tissue (lowering oxygen tension) [23] and expressed vascular endothelial growth factor [4], thus driving the early stages of heterotopic ossification. Furthermore, many of the novel correlations between the markers for brown fat, hypoxia, chondrogenesis, and ossification were present in the nodule region but not present in the normal fibrosa, indicating that these agents are likely relevant to nodule formation. The absence of these correlations from the prenodule region also suggests that differential remodeling is occurring between the smaller and larger nodules.

Correlations between brown fat-generated hypoxia and HA turnover were primarily present in the nodules and surrounding tissues, but generally absent in the normal-appearing fibrosa. Although more precise details regarding the interactions of these mechanisms cannot be determined using immunohistochemistry, we speculate that hypoxia in the nodule boundary (NodEdge) promotes HA synthesis. More towards the interior of the nodule (NodCtr), the action of hyaluronidases would generate HA fragments that may aid in promoting the differentiation of brown fat cells; the balance between HAS and hyaluronidase has been previously linked with the differentiation of adipocytes [37], as has TSG-6 [7]. Treatment of chondrocytes with low molecular weight HA (either directly or produced by hyaluronidases) induces a range of responses, including MMP synthesis [30], and HA promotes chondrogenic differentiation of adipose-derived stem cells [34]. Future investigations combining the application of a range of oxygen tensions to valve cells or to organ-cultured 
valve leaflets, with other experimental conditions that regulate HA turnover and induce chondrogenic and/or osteogenic differentiation should shed light on this novel mechanism.

\section{Role for HA regulation in calcification}

Our hypothesis that HA homeostasis could affect TSG-6 to either promote or inhibit BMP-2-mediated calcification appears to be supported by the presence of regionally specific correlations between HA degradation markers, ossification markers, and TSG-6 in PreNod, NodCtr, NodSurr, and PreNodSurr, and the strong correlations between HAS and these same brown fat and ossification markers in NodEdge. We speculate that when newly synthesized $\mathrm{HA}$ is abundant in a particular region of the valve tissue, as opposed being degraded by Hyal-1, HA will compete with BMP-2 for the same binding site on TSG-6, and as a result more BMP-2 will be free to promote calcification and growth of the nodule in this region. In the other regions where the expression of Hyal-1 is strong, the HA is more likely degraded and unable to bind to TSG-6; the binding of HA to the glycoprotein hyaluronectin, a hyaladherin, was previously reported to be size-dependent with smaller HA fragments (from 10 to $<60$ disaccharides) having lower binding affinity [2]. Absent or reduced binding between HA fragments and TSG-6 would thus allow the sequestration of BMP-2 and inhibit new calcification. Hyaluronidases have not been previously investigated in calcified aortic valves, but with regards to atherosclerosis their ability to generate low molecular weight HA has been linked with proliferation of smooth muscle cells (an effect also reported in valvular interstitial cells [18]) and VCAM-1 synthesis in vitro [3]. In three-dimensional in vitro cultures of cartilage slices, the addition of hyaluronidase promoted increased mineral accumulation [26]. HA itself appears to have a complicated relationship with calcification, with some reports suggesting that HA promotes the late osteogenic differentiation of cells [38] while others have integrated HA into biomaterial scaffolds and found either enhancement [15] or prevention [22, 25] of mineralization. Although there are no published investigations of the HA synthases in adult valves, the signaling pathways regulating the activity of HAS-1, HAS-2, and HAS-3 represent therapeutic targets for atherosclerosis [17] and potentially for CAVD as well. Further study of the dynamic synthesis and degradation of HA in heart valves, and how these mechanisms contribute to valve calcification in vitro and in vivo, will be necessary.

The results also showed positive expression of the HA binding receptors CD168 and HARE in NodSurr and PreNodSurr, respectively. This work was the first demonstration of CD168, also known as RHAMM (receptor for hyaluronic acid mediated motility) in heart valves. CD168, together with altered expression of HA and HA degradation, has also been linked with upregulation of angiogenesis in atherosclerosis [14] and wound healing [5]. For example, both CD168 and Hyal-1 were found in excess in complicated atherosclerotic plaques at risk of rupture, and the CD168 was particularly enhanced in neovessels within these regions. Thus, it has been proposed that the binding of degraded HA fragments to CD168 promotes endothelial cell activation and perpetuates angiogenic remodeling, key characteristics of atherosclerotic plaque hemorrhage and rupture, as well as valve calcification [14]. Our group has previously demonstrated the presence of HARE (HA receptor for endocytosis) in normal and myxomatous mitral valves [8], but its role in those valves as in these calcified valves is uncertain. In the kidney, HARE enables the clearance of HA and selected galactosaminoglycan proteoglycans from the bloodstream and is thought to be associated with HA turnover [35]. Interestingly, the distribution of CD44 was unremarkable and did not show profound correlations with valve calcification (data not shown), despite its well known roles in inflammatory mediation in atherosclerosis [3, 33]. CD44 was primarily involved with other HA regulatory mediators in the normal collagenous fibrosa. 
This work was also the first to demonstrate the presence of periostin in calcific valve nodules. Studies in young animal models have demonstrated that periostin regulates collagen fibrillogenesis and wound repair [9], the differentiation of mesenchymal cells towards a fibroblast phenotype [20], and the proper embryonic development and remodeling of heart valves [27]. In fact, periostin is absent from congenitally malformed bicuspid aortic valves, which lack the normal trilaminate structure [27]. Interestingly, periostin represses osteogenic differentiation programming of embryonic mesenchymal cells [31], although its isoform periostin-like-factor appears to promote osteogenic differentiation [36]. Although in this study periostin was strongly associated with markers for ossification (TSG-6), HA synthesis (HAS), hypoxia (HIF-1a) and brown adipocytic differentiation (PGC-1a), it is difficult to find other published reports of interactions between these proteins. Therefore, it will be important in the future to elucidate the function of periostin in adult aortic valve calcification, especially with regard to these other remodeling and differentiation mechanisms.

\section{Distinctions among nodule regions and prenodules}

The specificity and strength of the correlations between different mechanistic families was rather distinct between the inside and outside of the nodules, as well as between the nodules and prenodules. Taken together, these interactions between mechanistic families tended to occur in almost every region, although the specific markers involved tended to vary across the microanatomy. Correlations involving Hyal-1 were found in all nodule regions (NodCtr, PreNod) and surrounding tissues (NodSurr, PreNodSurr) except for NodEdge, where HAS and HIF-1a correlations were found. NodSurr had the greatest numbers of correlations between mechanistic families, which may be because more active cell-mediated remodeling was occurring within that region. PreNod and PreNodSurr were characterized by many very strong correlations between HA regulation and chondrogenesis, and ossification, but there were no interactions between these mechanisms and the hypoxic/brown fat mechanism, which suggests that prenodules are undergoing different remodeling than mature nodules and may represent an earlier stage of the nodule maturity. Alternative methods, such as the use of laser capture microdissection and gene chip analysis of mRNA isolated from these specific regions of calcified tissue sections, should offer considerable insight into the gene expression and signaling pathways involved in this highly localized remodeling.

\section{Limitations}

Although numerous correlations between different remodeling mechanisms were highly significant, the heterogeneous nature of the microstructural remodeling amongst the leaflet samples made it difficult to demonstrate significant differences in the abundance of the various markers through an analysis of variance. As a result, the work was primarily descriptive. As noted above, in the future, it may be useful to examine the interactions between these mechanisms in well controlled animal models, in vitro models, or in a larger series of human resected calcified valves stratified by clinical characteristics such as degree of aortic stenosis. It will also be important to clarify the roles of specific cell types or phenotypes in these mechanisms, as is has been demonstrated (and summarized by O'Brien [21]) that calcified heart valves contain activated valvular interstitial cells (expressing $a$ SMA, Fig. 2), valve cells undergoing chondrogenic or osteoblastic differentiation, various types of blood cells, and as shown here, cells expressing markers for brown fat differentiation.

\section{Conclusions}

This research demonstrated the first evidence that the proteins involved in regulation of HA turnover, as well as the promotion of hypoxic conditions by brown fat cells, are associated with ossification in calcific aortic stenosis. Additionally, the interactions between these 
mechanisms vary between large and small nodules and between the nodules and their surrounding tissues, with the tissues surrounding the nodules showing the greatest active remodeling. Further investigation of these regulatory and remodeling mechanisms, and their interactions with other pathways such as wnt, may offer insight into novel medical therapies that could intervene in numerous important pathological processes within the progression of this disease.

\section{Acknowledgments}

The authors thank the Rice University Institute for Biosciences and Bioengineering Medical Innovations Award for funding. Supported in part by NIH grants HL63090 (J.D.M.) and HL104377 (K.J.G.-A) and a Hertz Foundation Graduate Fellowship (E.H.S.).

\section{References}

1. Athanasou NA, Quinn J, Heryet A, Woods CG, McGee JO. Effect of decalcification agents on immunoreactivity of cellular antigens. J Clin Pathol. 1987; 40:874-878. [PubMed: 2443541]

2. Courel MN, Maingonnat C, Tranchepain F, Deschrevel B, Vincent JC, Bertrand P, Delpech B. Importance of hyaluronan length in a hyaladherin-based assay for hyaluronan. Anal Biochem. 2002; 302:285-290. [PubMed: 11878809]

3. Cuff CA, Kothapalli D, Azonobi I, Chun S, Zhang Y, Belkin R, Yeh C, Secreto A, Assoian RK, Rader DJ, Pure E. The adhesion receptor CD44 promotes atherosclerosis by mediating inflammatory cell recruitment and vascular cell activation. J Clin Invest. 2001; 108:1031-1040. [PubMed: 11581304]

4. Dilling CF, Wada A, Lazard Z, Salisbury E, Gannon F, Vadakkan T, Gao L, Hirschi K, Dickinson M, Davis AR, Olmsted-Davis E. Vessel formation is induced prior to the appearance of cartilage in BMP2-mediated heterotopic ossification. J Bone Miner Res. 2010; 25:1147-1156. [PubMed: 19839764]

5. Gao F, Yang CX, Mo W, Liu YW, He YQ. Hyaluronan oligosaccharides are potential stimulators to angiogenesis via RHAMM-mediated signal pathway in wound healing. Clin Invest Med. 2008; 31:E106-116. [PubMed: 18544273]

6. Grande-Allen KJ, Osman N, Ballinger ML, Dadlani H, Marasco S, Little PJ. Glycosaminoglycan synthesis and structure as targets for the prevention of calcific aortic valve disease. Cardiovasc Res. 2007; 76:19-28. [PubMed: 17560967]

7. Guo XR, Ding SL, Pan XQ, Gong HX, Fei L, Ni YH, Chen RH. Expression of TSG-6 gene during 3T3-11 preadipocyte differentiation and regulative role of tumor necrosis factor-alpha. Zhonghua Er Ke Za Zhi. 2004; 42:344-347. [PubMed: 15189690]

8. Gupta V, Barzilla JE, Mendez JS, Stephens EH, Lee EL, Collard CD, Laucirica R, Weigel PH, Grande-Allen KJ. Abundance and location of proteoglycans and hyaluronan within normal and myxomatous mitral valves. Cardiovasc Pathol. 2009; 18:191-197. [PubMed: 18621549]

9. Hamilton DW. Functional role of periostin in development and wound repair: Implications for connective tissue disease. J Cell Commun Signal. 2008; 2:9-17. [PubMed: 18642132]

10. Itoh S, Matubara M, Kawauchi T, Nakamura H, Yukitake S, Ichinose S, Shinomiya K. Enhancement of bone ingrowth in a titanium fiber mesh implant by rhBMP-2 and hyaluronic acid. J Mater Sci Mater Med. 2001; 12:575-581. [PubMed: 15348249]

11. Johansson B, Holmgren A, Hedstrom M, Engstrom-Laurent A, Engstrom KG. Evaluation of hyaluronan and calcifications in stenotic and regurgitant aortic valves. Eur J Cardiothorac Surg. 2011; 39:27-32. [PubMed: 20627752]

12. Katz R, Wong ND, Kronmal R, Takasu J, Shavelle DM, Probstfield JL, Bertoni AG, Budoff MJ, O'Brien KD. Features of the metabolic syndrome and diabetes mellitus as predictors of aortic valve calcification in the multi-ethnic study of atherosclerosis. Circulation. 2006; 113:2113-2119. [PubMed: 16636166]

13. Klewer SE, Yatskievych T, Pogreba K, Stevens MV, Antin PB, Camenisch TD. Has2 expression in heart forming regions is independent of bmp signaling. Gene Expr Patterns. 2006; 6:462-470.

[PubMed: 16458617] 
14. Krupinski J, Ethirajan P, Font MA, Turu MM, Gaffney J, Kumar P, Slevin M. Changes in hyaluronan metabolism and RHAMM receptor expression accompany formation of complicated carotid lesions and may be pro-angiogenic mediators of intimal neovessel growth. Biomark Insights. 2008; 2:361-367. [PubMed: 19662229]

15. Lisignoli G, Toneguzzi S, Zini N, Piacentini A, Cristino S, Tschon M, Grassi F, Fini M, Giardino R, Maraldi NM, Facchini A. Hyaluronan-based biomaterial (HYAFF-11) as scaffold to support mineralization of bone marrow stromal cells. Chir Organi Mov. 2003; 88:363-367. [PubMed: 15259552]

16. Lloyd-Jones D, Adams RJ, Brown TM, Carnethon M, Dai S, De Simone G, Ferguson TB, Ford E, Furie K, Gillespie C, Go A, Greenlund K, Haase N, Hailpern S, Ho PM, Howard V, Kissela B, Kittner S, Lackland D, Lisabeth L, Marelli A, McDermott MM, Meigs J, Mozaffarian D, Mussolino M, Nichol G, Roger VL, Rosamond W, Sacco R, Sorlie P, Roger VL, Thom T, Wasserthiel-Smoller S, Wong ND, Wylie-Rosett J. Heart disease and stroke statistics--2010 update: A report from the American Heart Association. Circulation. 2010; 121:e46-e215. [PubMed: 20019324]

17. Marzoll A, Nagy N, Wordehoff L, Dai G, Fries S, Lindner V, Grosser T, Fischer JW. Cyclooxygenase inhibitors repress vascular hyaluronan-synthesis in murine atherosclerosis and neointimal thickening. J Cell Mol Med. 2009; 13:3713-3719. [PubMed: 19320776]

18. Masters KS, Shah DN, Leinwand LA, Anseth KS. Crosslinked hyaluronan scaffolds as a biologically active carrier for valvular interstitial cells. Biomaterials. 2005; 26:2517-2525. [PubMed: 15585254]

19. Mohler ER 3rd, Chawla MK, Chang AW, Vyavahare N, Levy RJ, Graham L, Gannon FH. Identification and characterization of calcifying valve cells from human and canine aortic valves. J Heart Valve Dis. 1999; 8:254-260. [PubMed: 10399657]

20. Norris RA, Borg TK, Butcher JT, Baudino TA, Banerjee I, Markwald RR. Neonatal and adult cardiovascular pathophysiological remodeling and repair: Developmental role of periostin. Ann N Y Acad Sci. 2008; 1123:30-40. [PubMed: 18375575]

21. O'Brien KD. Pathogenesis of calcific aortic valve disease: A disease process comes of age (and a good deal more). Arterioscler Thromb Vasc Biol. 2006; 26:1721-1728. [PubMed: 16709942]

22. Ohri R, Hahn SK, Hoffman AS, Stayton PS, Giachelli CM. Hyaluronic acid grafting mitigates calcification of glutaraldehyde-fixed bovine pericardium. J Biomed Mater Res A. 2004; 70:328334. [PubMed: 15227678]

23. Olmsted-Davis E, Gannon FH, Ozen M, Ittmann MM, Gugala Z, Hipp JA, Moran KM, FouletierDilling CM, Schumara-Martin S, Lindsey RW, Heggeness MH, Brenner MK, Davis AR. Hypoxic adipocytes pattern early heterotopic bone formation. Am J Pathol. 2007; 170:620-632. [PubMed: 17255330]

24. Rodgers LS, Lalani S, Hardy KM, Xiang X, Broka D, Antin PB, Camenisch TD. Depolymerized hyaluronan induces vascular endothelial growth factor, a negative regulator of developmental epithelial-to-mesenchymal transformation. Circ Res. 2006; 99:583-589. [PubMed: 16931798]

25. Rodriguez KJ, Piechura LM, Masters KS. Regulation of valvular interstitial cell phenotype and function by hyaluronic acid in 2-D and 3-D culture environments. Matrix Biol. 2011; 30:70-82. [PubMed: 20884350]

26. Smetana K Jr, Stol M, Novak M. Artificial mineralization in vitro--a model of tissue mineralization. Folia Biol (Praha). 1993; 39:23-28. [PubMed: 8348982]

27. Snider P, Hinton RB, Moreno-Rodriguez RA, Wang J, Rogers R, Lindsley A, Li F, Ingram DA, Menick D, Field L, Firulli AB, Molkentin JD, Markwald R, Conway SJ. Periostin is required for maturation and extracellular matrix stabilization of noncardiomyocyte lineages of the heart. Circ Res. 2008; 102:752-760. [PubMed: 18296617]

28. Stephensand EH, Grande-Allen KJ. Age-related changes in collagen synthesis and turnover in porcine heart valves. J Heart Valve Dis. 2007; 16:672-682. [PubMed: 18095519]

29. Stephens EH, Saltarrelli JG, Baggett LS, Nandi I, Kuo JJ, Davis AR, Olmsted-Davis EA, Reardon MJ, Morrisett JD, Grande-Allen KJ. Differential proteoglycan and hyaluronan distribution in calcified aortic valves. Cardiovasc Pathol. 2011 in press. 
30. Stern R, Asari AA, Sugahara KN. Hyaluronan fragments: An information-rich system. Eur J Cell Biol. 2006; 85:699-715. [PubMed: 16822580]

31. Tkatchenko TV, Moreno-Rodriguez RA, Conway SJ, Molkentin JD, Markwald RR, Tkatchenko AV. Lack of periostin leads to suppression of notch1 signaling and calcific aortic valve disease. Physiol Genomics. 2009; 39:160-168. [PubMed: 19723774]

32. Tsukahara S, Ikeda R, Goto S, Yoshida K, Mitsumori R, Sakamoto Y, Tajima A, Yokoyama T, Toh S, Furukawa K, Inoue I. Tumour necrosis factor alpha-stimulated gene-6 inhibits osteoblastic differentiation of human mesenchymal stem cells induced by osteogenic differentiation medium and BMP-2. Biochem J. 2006; 398:595-603. [PubMed: 16771708]

33. Wilkinson TS, Bressler SL, Evanko SP, Braun KR, Wight TN. Overexpression of hyaluronan synthases alters vascular smooth muscle cell phenotype and promotes monocyte adhesion. J Cell Physiol. 2006; 206:378-385. [PubMed: 16110480]

34. Wu SC, Chang JK, Wang CK, Wang GJ, Ho ML. Enhancement of chondrogenesis of human adipose derived stem cells in a hyaluronan-enriched microenvironment. Biomaterials. 2010; 31:631-640. [PubMed: 19819543]

35. Zhou B, McGary CT, Weigel JA, Saxena A, Weigel PH. Purification and molecular identification of the human hyaluronan receptor for endocytosis. Glycobiology. 2003; 13:339-349. [PubMed: 12626425]

36. Zhu S, Barbe MF, Liu C, Hadjiargyrou M, Popoff SN, Rani S, Safadi FF, Litvin J. Periostin-likefactor in osteogenesis. J Cell Physiol. 2009; 218:584-592. [PubMed: 19006175]

37. Zizola CF, Julianelli V, Bertolesi G, Yanagishita M, Calvo JC. Role of versican and hyaluronan in the differentiation of 3T3-11 cells into preadipocytes and mature adipocytes. Matrix Biol. 2007; 26:419-430. [PubMed: 17513099]

38. Zou L, Zou X, Chen L, Li H, Mygind T, Kassem M, Bunger C. Effect of hyaluronan on osteogenic differentiation of porcine bone marrow stromal cells in vitro. J Orthop Res. 2008; 26:713-720. [PubMed: 18050326] 


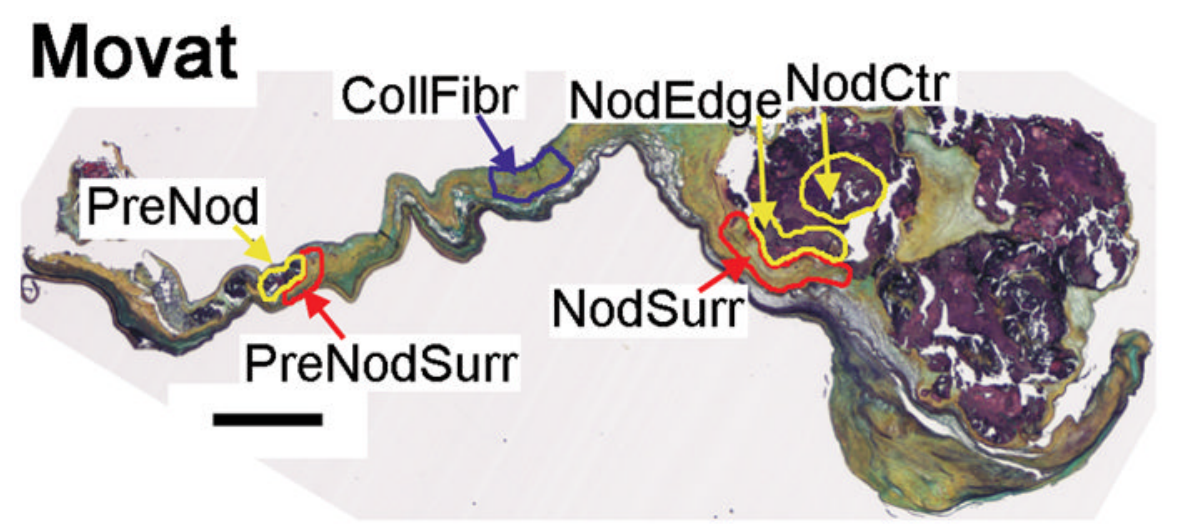

Fig. 1.

Movat pentachome stain of calcified aortic valve showing large nodule at the distal end and small prenodule more proximal to the annular edge of the leaflet. Scale bar indicates $1 \mathrm{~mm}$. Representative regions of analysis are outlined; these regions were small in some valves and larger in others based on the relative amount of soft vs. mineralized tissue. NodCtr: region within inner $2 / 3$ of large nodule. NodEdge: region within outer $1 / 3$ of large nodule. NodSurr = region surrounding large nodule. PreNod: small nodule. PreNodSurr = region surrounding small nodule. CollFibr: collagenous fibrosa within normal-appearing layered region of leaflet. 

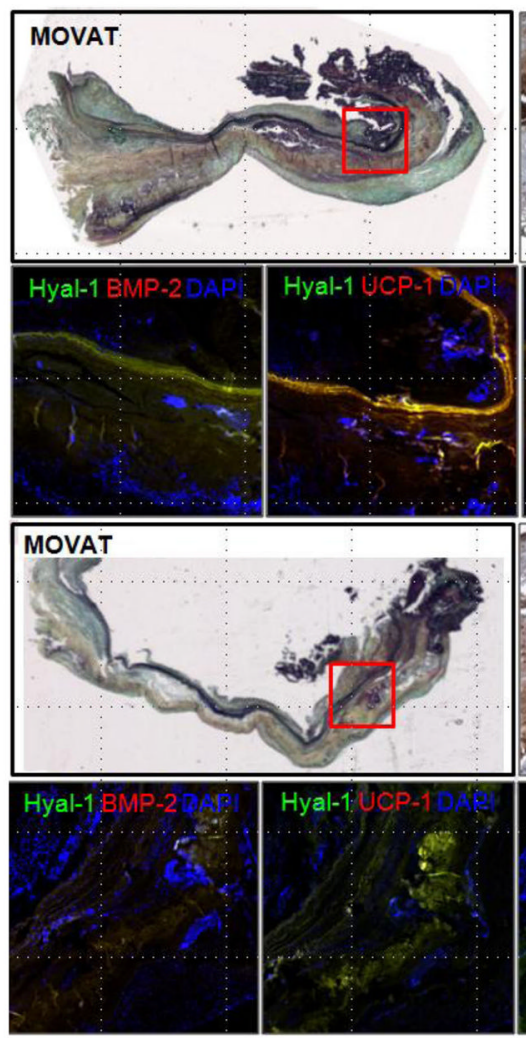

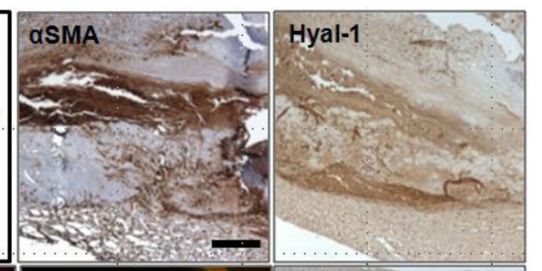

Hyal-1 TSG-6 6A
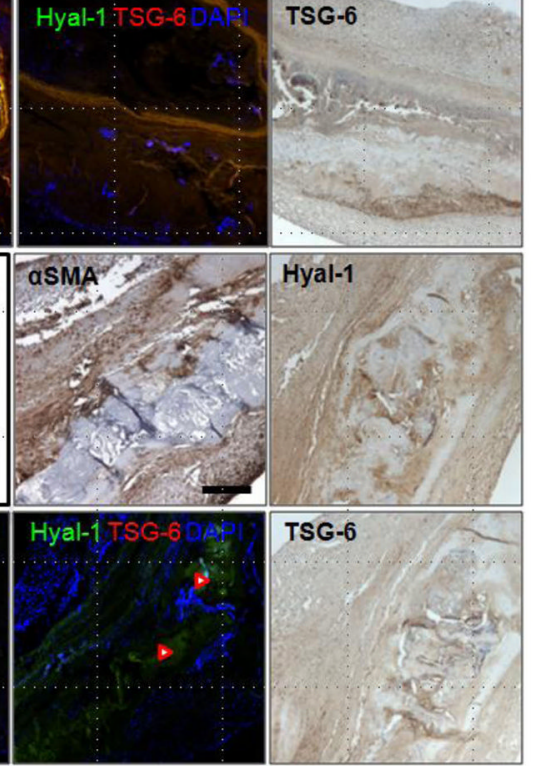

Fig. 2.

Movat pentachrome stained sections of calcified aortic valves and higher magnification views of immunostained sections of calcific nodule and surrounding tissue (indicated by red box on Movat stain). Images show the positive expression of aSMA, Hyal-1, and TSG-6 (in brown DAB chromagens) in the same region of tissue. Immunofluorescent double staining shows Hyal-1 (green) with BMP-2, UCP-1, or TSG-6 (red), and their colocalization (yellow color). Blue indicates cell nuclei. Red arrowheads indicate high TSG-6-Hyal-1 colocalization in the lower specimen. Scale bar $=100 \mu \mathrm{m}$. 

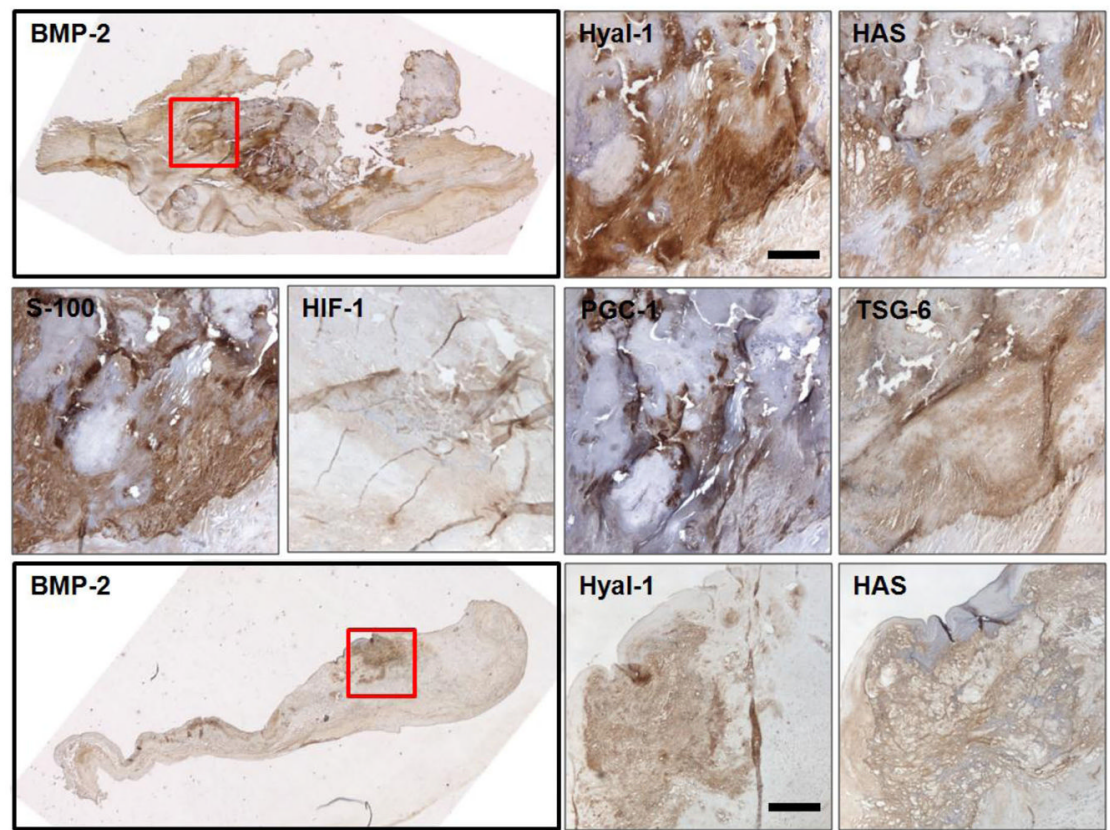

HAS
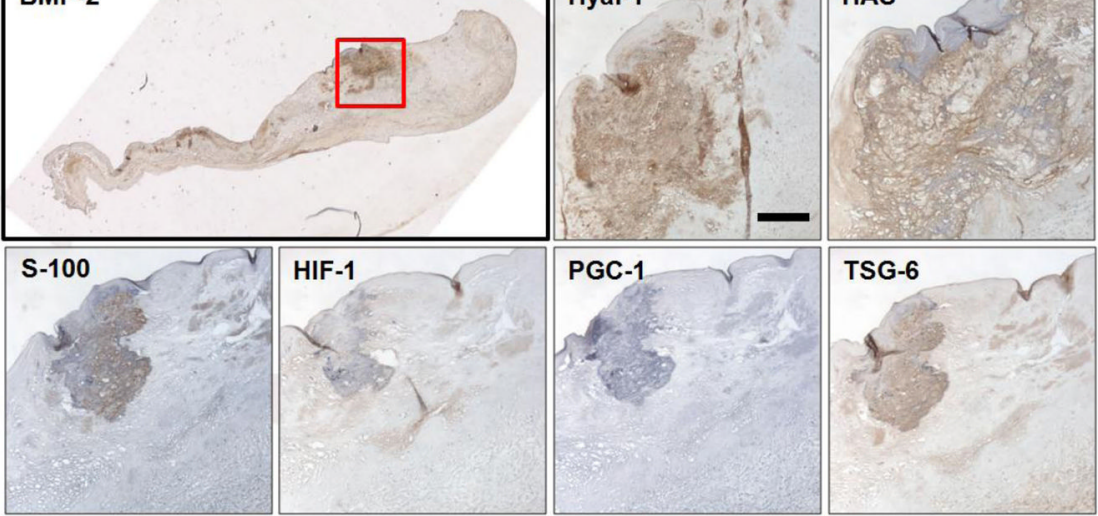

Fig. 3.

Immunohistochemically stained sections of calcified aortic valves shows expression (brown) of various antigens related to ossification (BMP-2, S-100, TSG-6), hyaluronan turnover (HAS, Hyal-1), hypoxia (HIF-1), and brown adipocyte differentiation (PGC-1) in highly calcified regions of diseased aortic valves. Higher magnification images are from the region outlined in red on the BMP-2 immunostaining image. Scale bar $=100 \mu \mathrm{m}$. 

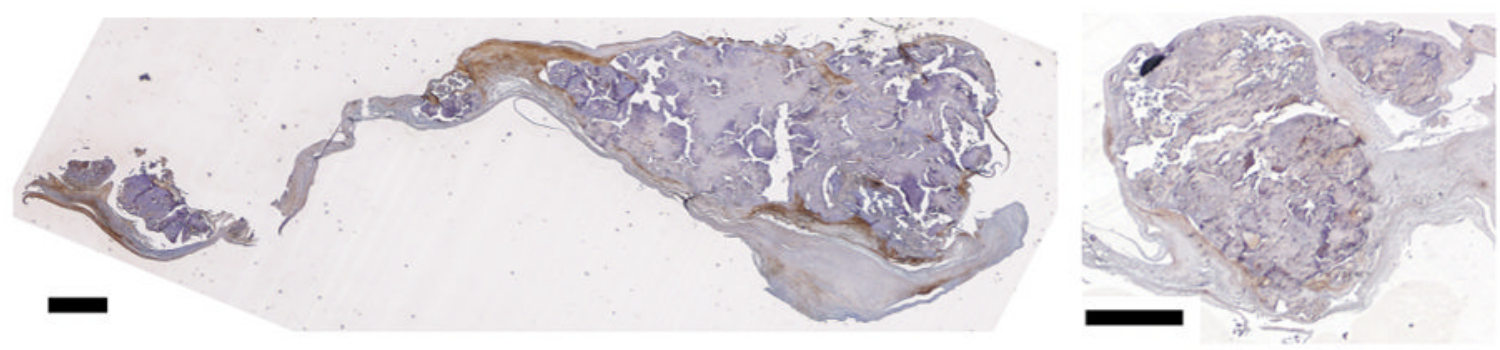

Fig. 4.

Periostin staining relative to nodules in calcified aortic valves demonstrating stronger staining in the center of and at the edge of nodules relative to surrounding tissues. Scale bars indicate $1 \mathrm{~mm}$. 
A BMP-2 and TSG-6 in Nodule Surrounding
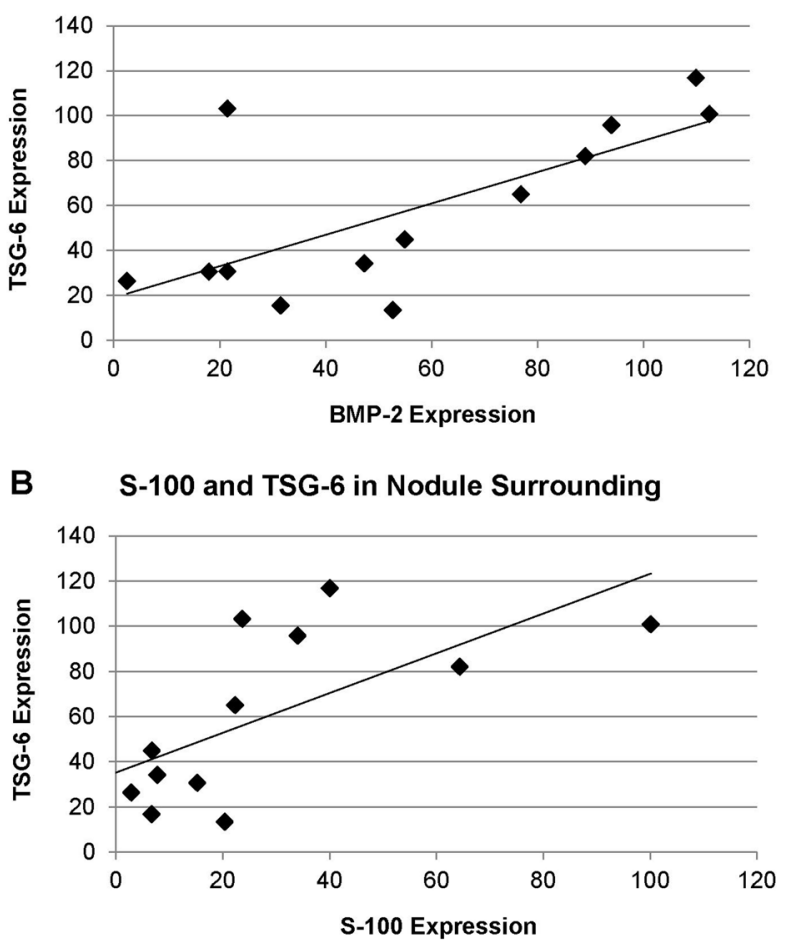

Fig. 5.

Correlations between expression of TSG-6 and (A) BMP-2 and (B) S-100 in the tissue surrounding the large calcific nodules (NodSurr). Linear fits correspond to correlations shown in Table 2. 
A

Hyal-1 and UCP-1 Correlations

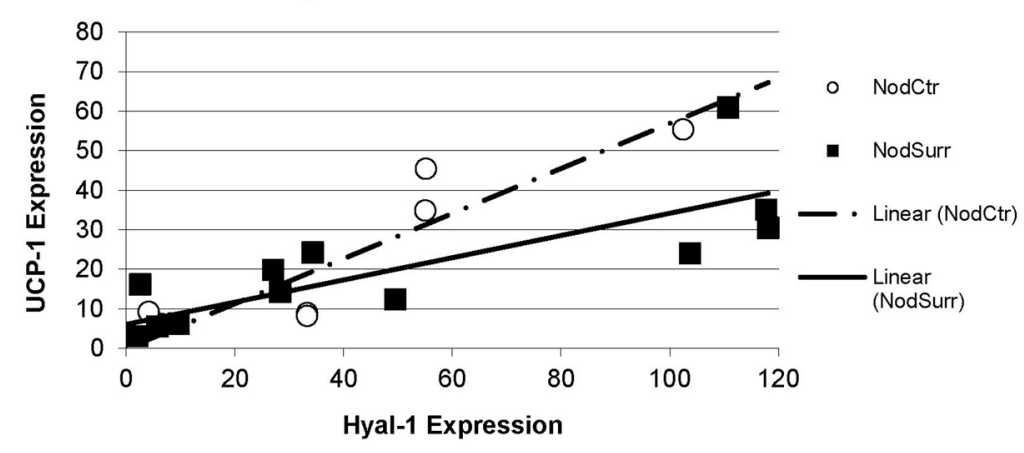

B

Hyal-1 and TSG-6 Correlations

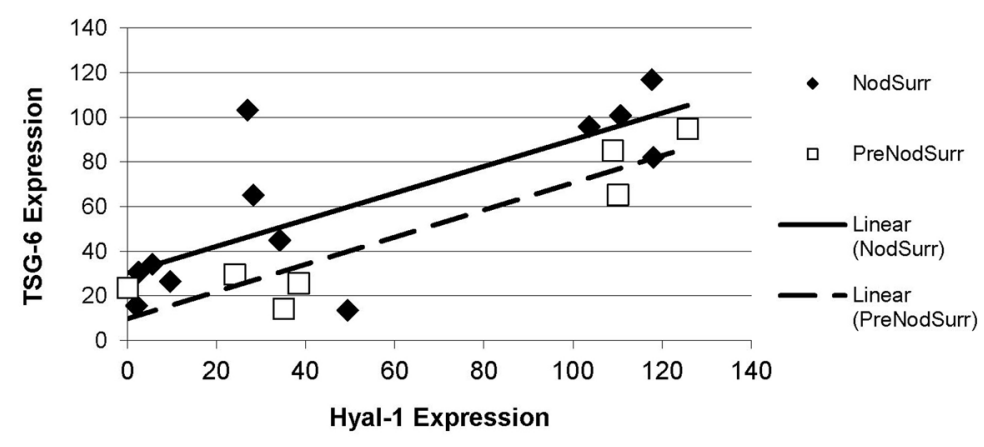

Fig. 6.

(A) Correlations between expression of Hyal-1 and UCP-1 in the center of the large calcific nodules (NodCtr) and in the tissue surrounding the large calcific nodules (NodSurr). (B) Correlations between expression of Hyal-1 and TSG-6 in the tissue surrounding the large calcific nodules (NodSurr) and surrounding the small calcific nodulues (PreNodSurr). Linear fits correspond to correlations shown in Table 3 . 


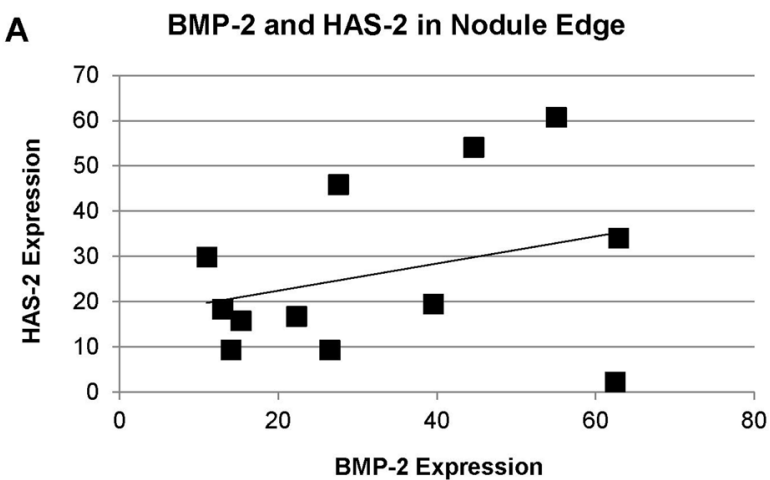

B S-100 and UCP-1 in Nodule Edge

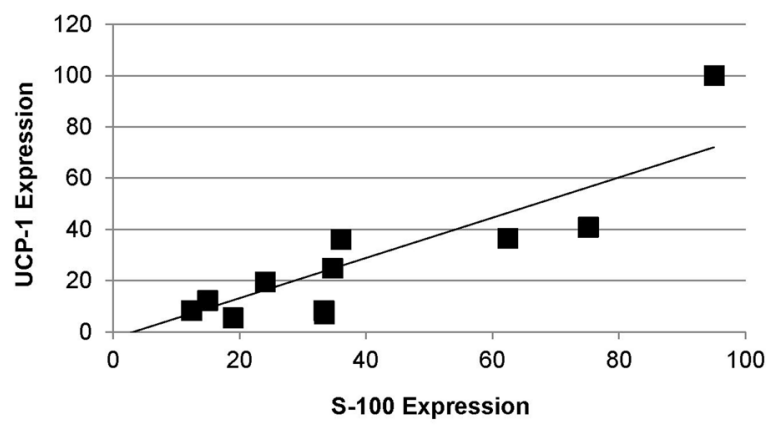

C BMP-2 and Hyal-1 in Pre-Nodule

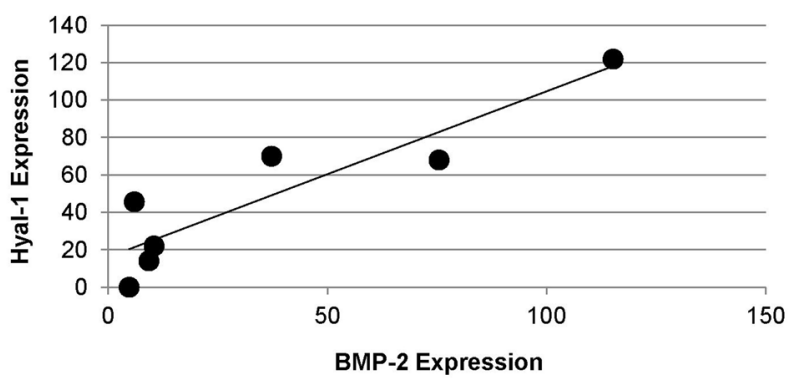

Fig. 7.

Representative correlations in the edge of the large calcified nodules (NodEdge): (A) BMP-2 and HAS-2; (B) S-100 and UCP-1. (C) Correlation between BMP-2 and Hyal-1 in the interior of the small calcified nodules (PreNod). Linear fits correspond to correlations shown in Table 3. 
Table 1

Antibodies Used in Immunohistochemistry.

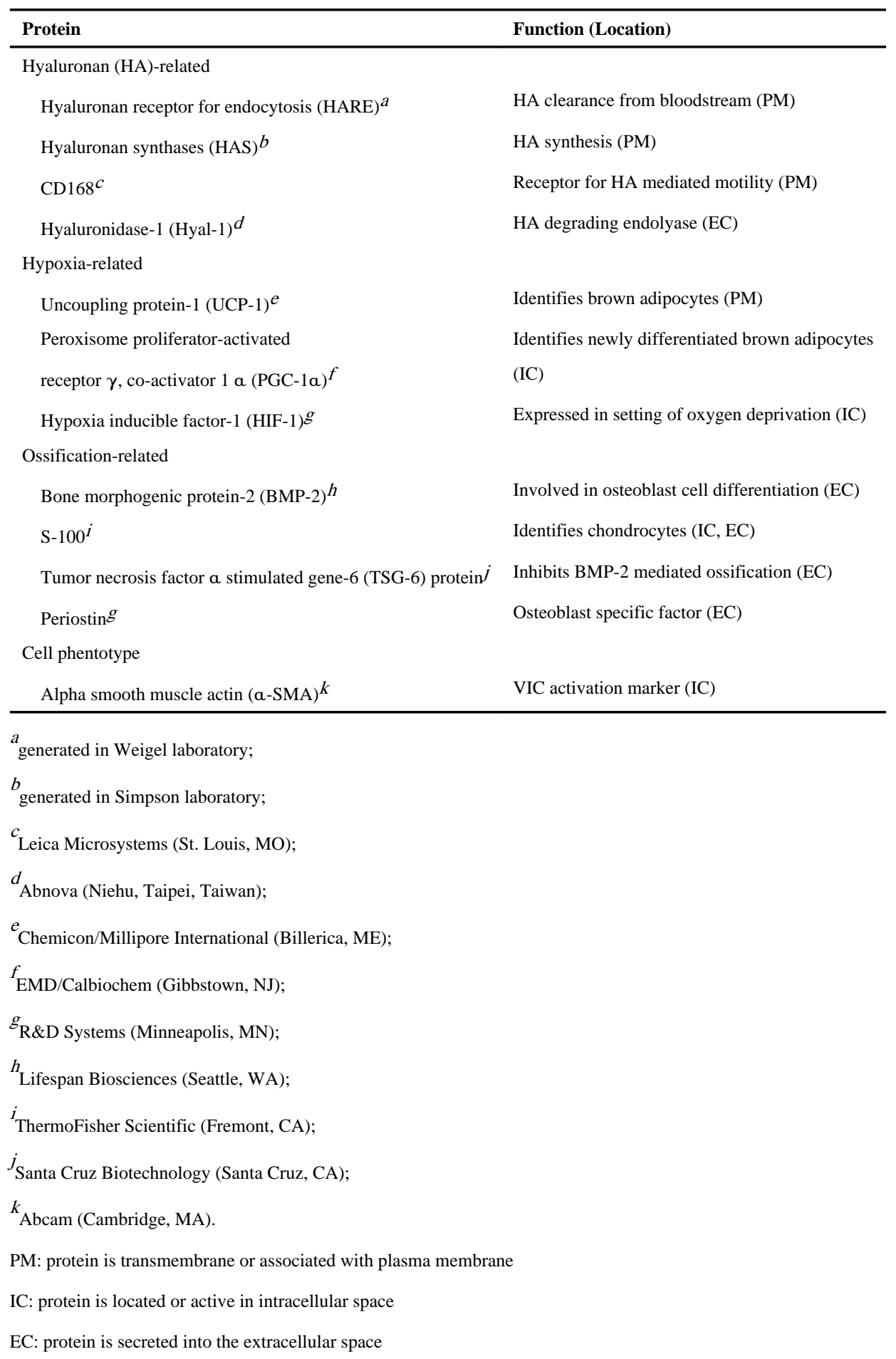


Table 2

Correlations within Mechanistic Families.

\begin{tabular}{ccccc}
\hline Marker 1 & Marker 2 & Region & R & $\boldsymbol{P}$ \\
\hline Hyaluronan Regulation & & & \\
CD168 & Hyal-1 & NodSurr & 0.889 & $<0.0001$ \\
CD168 & Hyal-1 & CollFibr & 0.596 & 0.0091 \\
HARE & Hyal-1 & CollFibr & 0.798 & $<0.0001$ \\
Hypoxia & & & & \\
HIF-1a & UCP-1 & NodSurr & 0.631 & 0.0050 \\
HIF-1a & PGC-1a & NodEdge & 0.849 & $<0.0001$ \\
HIF-1a & UCP-1 & PreNodSurr & 0.880 & 0.0039 \\
PGC-1a & UCP-1 & NodCtr & 0.721 & 0.0024 \\
Endochondral Ossification & & & \\
BMP-2 & S-100 & NodSurr & 0.716 & 0.0008 \\
BMP-2 & TSG-6 & NodSurr & 0.668 & 0.0025 \\
BMP-2 & TSG-6 & PreNodSurr & 0.873 & 0.0102 \\
S-100 & TSG-6 & NodSurr & 0.674 & 0.0022 \\
S-100 & TSG-6 & NodEdge & 0.585 & 0.0086 \\
Periostin & TSG-6 & NodCtr & 0.856 & 0.0008 \\
\hline
\end{tabular}

NodSurr=tissue surrounding nodule, NodEdge=edge of nodule, PreNodSurr=tissue surrounding prenodule, NodCtr=center of nodule, CollFibr=normal appearing fibrosa far from nodules.

Non-italicized p-values are significant correlations $(P<0.0045)$.

Italicized p-values are trends $(P \unlhd) .01)$. 


\section{Table 3}

Correlations Between Different Mechanistic Families

\begin{tabular}{|c|c|c|c|c|}
\hline Region & Marker 1 & Marker 2 & $\mathbf{r}$ & $\boldsymbol{P}$ \\
\hline \multicolumn{5}{|l|}{ NodSurr } \\
\hline & BMP-2 & Hyal-1 & 0.772 & 0.0002 \\
\hline & & CD168 & 0.695 & 0.0014 \\
\hline & & UCP-1 & 0.608 & 0.0075 \\
\hline & CD168 & S-100 & 0.797 & 0.0001 \\
\hline & & TSG-6 & 0.741 & 0.0004 \\
\hline & & UCP-1 & 0.662 & 0.0028 \\
\hline & Hyal-1 & S-100 & 0.813 & $<0.0001$ \\
\hline & & UCP-1 & 0.716 & 0.0008 \\
\hline & & TSG-6 & 0.700 & 0.0012 \\
\hline & $S-100$ & UCP-1 & 0.672 & 0.0022 \\
\hline & TSG-6 & UCP-1 & 0.634 & 0.0047 \\
\hline \multicolumn{5}{|c|}{ NodEdge (Outer $1 / 3$ ) } \\
\hline & BMP-2 & HAS & 0.587 & 0.0083 \\
\hline & HAS & HIF-1a & 0.730 & 0.0004 \\
\hline & & PGC-1a & 0.744 & 0.0004 \\
\hline & & Periostin & 0.702 & 0.0051 \\
\hline & HIF-1a & Periostin & 0.667 & 0.0092 \\
\hline & PGC-1a & Periostin & 0.693 & 0.0086 \\
\hline & S-100 & UCP-1 & 0.662 & 0.0020 \\
\hline \multicolumn{5}{|c|}{ NodCtr (I nner 1/3) } \\
\hline & Hyal-1 & UCP-1 & 0.900 & $<0.0001$ \\
\hline & & PGC-1a & 0.734 & 0.0019 \\
\hline & & S-100 & 0.641 & 0.0075 \\
\hline & S-100 & UCP-1 & 0.704 & 0.0023 \\
\hline & BMP-2 & PGC-1a & 0.701 & 0.0036 \\
\hline \multicolumn{5}{|l|}{ PreNod } \\
\hline & BMP-2 & Hyal-1 & 0.912 & 0.0042 \\
\hline & HARE & S-100 & 0.902 & 0.0055 \\
\hline & Hyal-1 & S-100 & 0.932 & 0.0023 \\
\hline & & TSG-6 & 0.922 & 0.0089 \\
\hline \multicolumn{5}{|c|}{ PreNodSurr } \\
\hline & Hyal-1 & BMP-2 & 0.937 & 0.0018 \\
\hline & & TSG-6 & 0.957 & 0.0007 \\
\hline \multicolumn{5}{|l|}{ CollFibr } \\
\hline & UCP-1 & CD168 & 0.673 & 0.0022 \\
\hline & & Hyal-1 & 0.743 & 0.0003 \\
\hline & & S-100 & 0.612 & 0.0069 \\
\hline & HARE & S-100 & 0.666 & 0.0035 \\
\hline
\end{tabular}

Pathol Res Pract. Author manuscript; available in PMC 2013 November 15. 


\begin{tabular}{ccccc}
\hline Region & Marker 1 & Marker 2 & r & $\boldsymbol{P}$ \\
\hline & PGC-1 & Periostin & 0.761 & 0.0010 \\
\hline
\end{tabular}

NodSurr=tissue surrounding nodule, NodEdge=edge of nodule, NodCtr=center of nodule, PreNod=center of prenodule, PreNodSurr=tissue surrounding prenodule, CollFibr=normal appearing fibrosa far from nodules.

Non-italicized p-values are significant correlations $(P<0.0045)$.

Italicized p-values are trends $(P \unlhd) .01)$. 